\title{
Sonographic Features and its Association with Visual Outcome and Microbiological Profile in Acute Post Cataract Surgery Endophthalmitis - A Prospective Analysis
}

\author{
Tharini Senthamizh ${ }^{1 *}$, Tanpreet Pal Singh ${ }^{2}$ and Haripriya Aravind ${ }^{3}$ \\ ${ }^{1}$ Resident, Aravind Eye Hospital, Madurai, India \\ ${ }^{2}$ Medical Officer, Department of Cataract and IOL Services, Aravind Eye Hospital, \\ Madurai, India \\ ${ }^{3}$ Professor and Head, Department of Cataract and IOL Services, Aravind Eye \\ Hospital, Madurai, India \\ *Corresponding Author: Tharini Senthamizh, Resident, Aravind Eye Hospital, \\ Madurai, India.
}

Received: September 06, 2020

Published: September 28, 2020

(C) All rights are reserved by Tharini

Senthamizh., et al.

\begin{abstract}
Background and Aims: Cataract surgery is the commonest eye surgery performed worldwide. Endophthalmitis is a sight threatening complication of cataract surgery which causes reduced vitreous clarity and media opacities and precludes visualization of the posterior segment. Ultrasonography is done in cases of suspected endophthalmitis with poor media clarity. This study aims at evaluating, baseline sonographic features of acute post-cataract surgery endophthalmitis and its association with initial vision, final visual outcome and microbiological profile.

Materials and Methods: This prospective study conducted at Aravind Eye Hospital, Madurai, South India, included all patients who presented with clinical symptoms and signs of endophthalmitis, between January 2014 to December 2015. They underwent A-mode and B-mode ultrasonography and were managed with either 'tap and inject' strategy or primary vitrectomy and followed up for three months. The sonographic features like vitreous opacities, vitreous membranes, retinal detachment, choroidal detachment, posterior vitreous detachment, and retino-choroidal thickening were documented and their association with vision at presentation, microbiological profile and final visual acuity was studied.

Results: There were 54 patients (54 eyes), their mean age was 59.9 \pm 19.2 years and male : female ratio was 1:1. Of them, 33 patients (61.1\%) had dense vitreous opacities, 16 patients (29.6\%) had dense vitreous membrane, 3 patients (5.6\%) had retinal detachment and 1 patient (1.9\%) had choroidal detachment. Median retino-choroidal thickening was found to be $1.82 \mathrm{~mm}$ (1.67-1.96 mm). Increased retino-choroidal thickening $(\mathrm{P}=0.049)$ and dense vitreous opacities $(\mathrm{P}=0.000)$ was significantly associated with poor initial presenting acuity. The occurrence of retinal detachment was significantly associated with poor final vision $(\mathrm{P}=0.006)$ and infection by virulent organisms $(\mathrm{P}=0.028)$.

Conclusion: In conclusion, sonographic warning signs are associated with infection by virulent organisms and poor visual outcome.
\end{abstract} Keywords: Cataract; Endophthalmitis; Ultrasonography; Vitrectomy; Visual Acuity

\section{Abbreviations}

RD: Retinal Detachment; CD: Choroidal Detachment; PVD: Posterior Vitreous Detachment; RCT: Retino-Choroidal Thickening

\section{Introduction}

Endophthalmitis is a potentially sight threatening complication following ocular surgery, trauma to the eye and endogenous infections. Owing to the increasing number of ocular procedures every year, post-operative endophthalmitis is on the rise [1]. The incidence of post cataract surgery endophthalmitis in India has been reported to range from $0.02 \%$ to $0.09 \%$, whereas worldwide incidence ranges from $0.02 \%$ to $0.26 \%$ [2]. Endophthalmitis is an inflammation of the inner coats of the eyeball, thereby presents 
with reduced vitreous clarity and media opacities, which prevent visualization of posterior segment. There may be associated retinal or choroidal detachment in such patients, which may not be visible on routine fundus examination. Ultrasonography serves as a safe and readily available modality to aid in the diagnosis of infectious endophthalmitis. It is invariably done in all cases of suspected endophthalmitis with poor media clarity [3]. There are few studies correlating the sonographic features and the final visual outcome, but the results are inconclusive and the features reported are not specific for acute post cataract surgery endophthalmitis $[4,5]$.

\section{Aim of the Study}

Hence, this study aims at evaluating baseline sonographic features in acute post-operative endophthalmitis and its association with vision at presentation, final visual outcome, and microbiological profile.

\section{Materials and Methods}

This prospective observational study was conducted in Aravind Eye Hospital, Madurai, South India, from January 2014 to December 2015, after approval of Institutional Review Board. All patients who presented with symptoms of redness, pain, and diminution of vision within 6 weeks following cataract surgery, both operated in-house and in other centers, were included in our study. Endophthalmitis following other ocular procedures, trauma and endogenous causes were excluded. Those who presented with corneal ulcers and opacities for whom vitrectomy could not be performed, were also excluded from our study. All patients were admitted and their baseline data including age, postoperative day of presentation, and symptoms were documented. Visual acuity was recorded using Snellen's visual acuity chart. Anterior segment examination and fundus examination was done using slit lamp biomicroscopy and indirect ophthalmoscopy, respectively. Combined A-scan and B-scan (Ultrascan, Alcon) was performed for all included patients, by two expert retina physicians. Sonographic features documented were vitreous opacities and vitreous membranes graded as none, mild, moderate, and dense; retinal detachment (RD), choroidal detachment (CD), posterior vitreous detachment (PVD), and degree of retino-choroidal thickening (RCT), graded as present or absent.

The decision of initial vitreous tap and injection of intravitreal antibiotics, or vitrectomy was left to the discretion of the treating surgeon. Intraocular samples for microbiological analysis were collected during anterior chamber tap, vitreous tap or during vitrectomy. All samples were inoculated immediately and sent for gram stain, $\mathrm{KOH}$ stain and cultured in blood agar, Sabouraud's agar, Thioglycolate broth and Brain heart infusion broth. No transport media was used. Those who worsened with conservative management underwent salvage core vitrectomy. Snellen's visual acuity of all patients was documented at discharge, at one month and at 3 months follow up. A standard logMAR scale was used for statistical analysis. LogMAR Visual acuity is described as follows: no light perception - 4.0, perception of light - 3.0, Hand movements - 2.6 and finger counting - 2.3 [6]. Continuous variables like age, time of presentation after surgery, initial vision, final vision and retinochoroidal thickness were expressed as mean with standard deviation or median with interquartile range based on their normality of distribution. Categorical variables like gender, hypopyon, fibrillary membrane, vitreous opacities, vitreous membranes, $R D, C D$, PVD were expressed in percentage. Change in visual acuity was defined as one line increment or decrement from presenting vision at 3 months follow-up. The association between sonographic findings and presenting vision, microbiological profile, final vision and change in vision was analyzed using Kruskal Wallis test or Fisher's exact test depending on the number of subgroups in each category. P-value of less than 0.05 was considered as statistically significant.

\section{Results}

We had 54 patients (54 eyes) with acute post cataract surgery endophthalmitis, of whom 27 were males and 27 were females. Their mean age was $59.9 \pm 9.6$ years. The median duration of presentation after cataract surgery was 14.5 days (2 to 55 days). 26 patients (48.1\%) had hypopyon at presentation and 41 patients (75.9\%) had fibrillary membrane over the intraocular lens. Red reflex was absent in 44 patients $(81.5 \%)$ and faint red glow was present in 9 patients (16.7\%). B-scan ultrasound showed vitreous echogenicity in all the patients. Retinal detachment and choroidal detachment were present in 3 patients $(5.6 \%)$ and 1 patient (1.9\%) respectively. Median retinochoroidal thickening was found to be $1.82 \mathrm{~mm}$ (1.67 to $1.96 \mathrm{~mm}$ ). Initial management done was vitreous or aqueous tap and intravitreal antibiotics in 42 patients (77.8\%), primary vitrectomy in 24 patients (44.4\%) (Table 1). At third month, 47 patients $(87 \%)$ had improvement in vision and 3 patients (5.6\%) had decrement in vision.

\begin{tabular}{|l|c|}
\hline Presentation & $\begin{array}{c}\text { Median with IQR } \\
\text { (\%) }\end{array}$ \\
\hline Presenting visual acuity in log MAR & $2.3(1.02-2.6)$ \\
\hline Post-operative day of presentation & $14.5(7.0-22.2)$ \\
\hline Initial management & \\
a) Tap and inject* & $42(77.8 \%)$ \\
b) Vitrectomy* & $24(44.4 \%)$ \\
\hline Final visual acuity in log MAR & $0.3(0.2-0.8)$ \\
\hline
\end{tabular}

Table 1: Clinical characteristics, initial treatment, and final outcome.

\#: Median with Interquartile range.

*: Number (percentage). 


\section{Microbiological profile}

Among the 54 samples (54 eyes) for microbiological analysis, $41(75.9 \%)$ were culture negative, 8 (14.8\%) had grown Coagulase Negative Staphylococcus (CONS), 2 (3.7\%) had Staphylococcus aureus, 1 (1.8\%) had Streptococci and 1 (1.8\%) had Pseudomonas growth. The sonographic features found in these patients include vitreous debris, vitreous membranes, retinal detachment, choroidal detachment, total or incomplete posterior vitreous detachment and increased retino-choroidal thickening. Due to small number of positive isolates, we classified the microbiological profile as culture negative, CONS, and virulent organism group (which included Staphylococcus aureus, Streptococci and Pseudomonas) [7]. The sonographic features of all the three groups were analyzed separately (Table 2). Mean final visual acuity was $0.4 \log$ MAR in the culture negative group, $0.3 \log$ MAR in the CONS group and $2.3 \log$ MAR in the virulent organism group. Among the 8 patients in CONS group, $6(75 \%)$ had dense vitreous opacities, $3(37.5 \%)$ had dense vitre- ous membranes and none of them had retinal detachment, choroidal detachment, or posterior vitreous detachment. Dense vitreous opacities and dense vitreous membranes were also more in virulent organism group when compared to the other groups. On comparing the three groups with vitreous opacities as none, mild and moderate and dense, no significant association was found $(\mathrm{P}=$ 0.621 , Fischer's exact test). We did not find any significant association between three culture groups and vitreous membranes graded as none, mild and moderate and dense $(\mathrm{P}=1.000$, Fischer's exact test). However, presence of retinal detachment had significant association with virulent organism group. Of the three patients who had retinal detachment, two (66.6\%) belonged to virulent organism group and one $(33.3 \%)$ to culture negative group $(\mathrm{P}=0.028$, chi square test). Also, there was a statistically significant correlation between retino-choroidal thickening and virulent organism group ( $\mathrm{P}=0.009$, Kruskal Wallis test). Other findings like CD and PVD had no association with the microbiological profile.

\begin{tabular}{|c|c|c|c|}
\hline Sonographic features & $\begin{array}{c}\text { Culture negative } \\
\text { n (\%) }\end{array}$ & $\begin{array}{l}\text { CONS } \\
\mathrm{n}(\%)\end{array}$ & $\begin{array}{c}\text { Virulent organisms* } \\
\text { n (\%) }\end{array}$ \\
\hline \multicolumn{4}{|l|}{ Vitreous opacities } \\
\hline Dense & $23(56.1 \%)$ & $6(75 \%)$ & $4(80 \%)$ \\
\hline Mild and Moderate & $17(41.5 \%)$ & $2(25 \%)$ & $1(20 \%)$ \\
\hline None & $1(2.4 \%)$ & $0(0)$ & $0(0)$ \\
\hline \multicolumn{4}{|l|}{ Vitreous membranes } \\
\hline Dense & $12(29.3 \%)$ & $3(37.5 \%)$ & $2(40 \%)$ \\
\hline Mild and Moderate & $13(31.7 \%)$ & $2(25 \%)$ & $1(20 \%)$ \\
\hline None & $16(39 \%)$ & $3(37.5 \%)$ & $2(40 \%)$ \\
\hline \multicolumn{4}{|l|}{ Retinal detachment } \\
\hline Absent & $40(97.6 \%)$ & $8(100 \%)$ & $3(60 \%)$ \\
\hline Present & $1(2.4 \%)$ & $0(0)$ & $2(40 \%)$ \\
\hline \multicolumn{4}{|l|}{ Choroidal detachment } \\
\hline Absent & $40(97.6 \%)$ & $8(100 \%)$ & $5(100 \%)$ \\
\hline Present & $1(2.4 \%)$ & $0(0)$ & $0(0)$ \\
\hline \multicolumn{4}{|l|}{ PVD $^{\#}$} \\
\hline Absent & $34(82.9 \%)$ & $8(100 \%)$ & $5(100 \%)$ \\
\hline Present & $7(17.1 \%)$ & $0(0)$ & $0(0)$ \\
\hline
\end{tabular}

Table 2: Sonographic features in three groups of microbiological culture.

CONS: Coagulase Negative staphylococci.

*: Virulent organisms -Staphylococcus, Streptococcus, and Pseudomonas.

\#: PVD-Posterior Vitreous Detachment. 
Vision at presentation, final visual outcome, and improvement in the vision

The median vision at presentation was $2.3 \log$ MAR $(0.2$ to 4.0$)$ and visual acuity at presentation had a statistically significant association with dense vitreous opacities ( $\mathrm{P}=0.000$, Kruskal Wallis test). Also, retino-choroidal thickening had a statistically significant correlation with presenting visual acuity $(\mathrm{P}=0.049$, Spearman's correlation). Of the 54 eyes, improvement in vision was noted in 50 patients $(92.5 \%)$ and in remaining 4 (7.5\%), vision remained unchanged at 3 months. There was a statistically significant association between improvement in vision and absence of retinal detachment (P-0.04, Fischer's exact test). None of the other ultrasonographic features were associated with change in vision at three months (Table 3). Among 33 patients with dense vitreous opacities, 31 patients (93.9\%) had improvement in vision, 1 patient $(3 \%)$ had decreased vision and vision in the other 1 patient (3\%) was static at third month. 15 out of 17 patients (88.2\%) with dense vitreous membranes had an improvement in vision and the other two remained static (11.8\%). We had only one patient with choroidal detachment who showed improvement in vision. The median final vision at 3 months was $0.3 \log$ MAR ( 0.0 to $4.0 \log$ MAR). Final visual acuity had a statistically significant association with the presence of retinal detachment, ( $\mathrm{P}=0.006$, Fischer's exact test) but other sonographic features had no impact on the final visual outcome. Also, a statistically significant difference was noted between the groups with and without retinal detachment in B-scan on comparing their mean final visual acuity (RD present- $2.7 \log$ MAR vs RD absent- 0.4 $\log$ MAR, $\mathrm{P}<0.01$, $\mathrm{t}$-test). Mean final visual acuity of all the sonographic categories are depicted in figure 1 and 2.

\begin{tabular}{|l|c|c|c|c|}
\hline Sonographic feature & $\begin{array}{c}\text { Presenting Vision } \\
\text { (logMAR) }\end{array}$ & $\begin{array}{c}\text { Final Vision } \\
\text { (logMAR) }\end{array}$ & $\begin{array}{c}\text { Change in vision } \\
\text { (logMAR) }\end{array}$ & $\begin{array}{c}\text { Micro-organisms isolated in } \\
\text { culture p-value }\end{array}$ \\
\hline Vitreous opacities & $0.000^{\mathrm{a}}$ & $0.508^{\mathrm{a}}$ & $0.236^{*}$ & $0.621^{*}$ \\
\hline Vitreous membranes & $0.278^{\mathrm{a}}$ & $0.865^{\mathrm{a}}$ & $0.619^{*}$ & $1.000^{*}$ \\
\hline Retinal detachment & $0.118^{*}$ & $0.006^{*}$ & $0.041^{*}$ & $0.028^{*}$ \\
\hline Choroidal detachment & $0.948^{*}$ & $0.296^{*}$ & $1.000^{*}$ & $1.000^{*}$ \\
\hline Posterior vitreous detachment & $0.062^{*}$ & $0.576^{*}$ & $0.401^{*}$ & $0.628^{*}$ \\
\hline Retino-choroidal thickening & $0.049^{\#}$ & $0.342^{\#}$ & $0.196^{\mathrm{a}}$ & $0.009^{\mathrm{a}}$ \\
\hline
\end{tabular}

Table 3: Correlation of ultrasonographic findings with visual acuity and microbiological profile.

a: Kruskal Wallis test.

\#: Spearman's correlation test.

*: Fischer exact test.

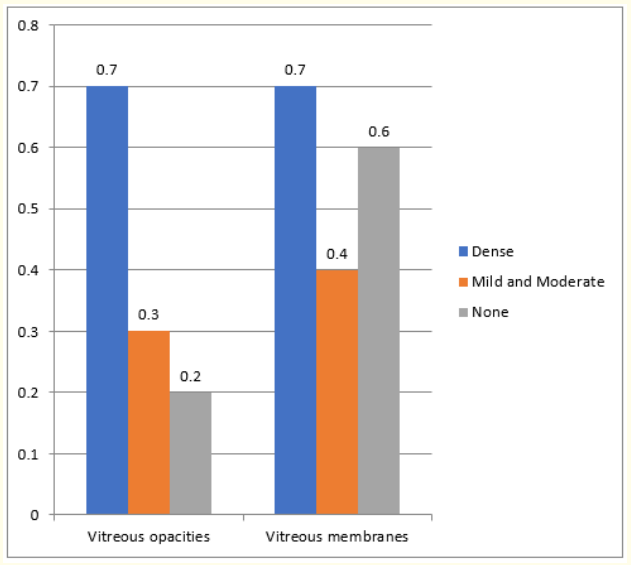

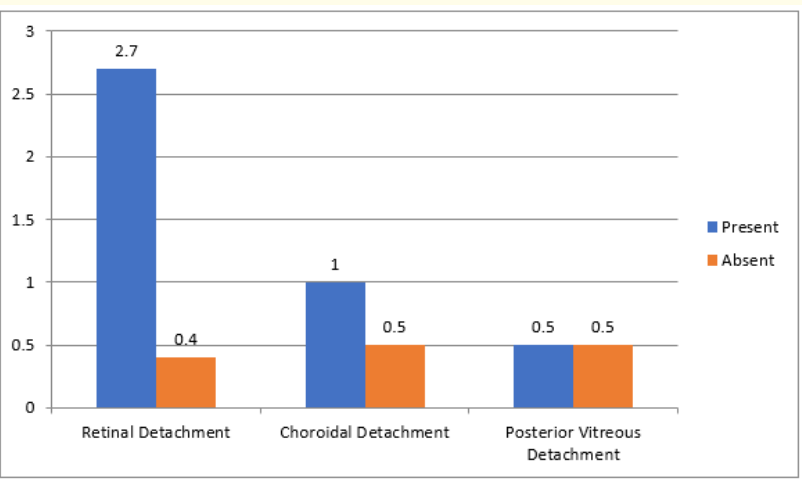

Figure 2: Mean final visual acuity of RD, CD and PVD.

Figure 1: Comparison of Mean final visual acuity between the three groups of vitreous opacities and vitreous membranes. 


\section{Discussion}

Endophthalmitis following cataract surgery, is a serious complication that may lead to visual loss. The visual outcome depends on virulence of organisms, disease severity and timing of intervention [8]. Clinical evaluation is often limited by media opacities, in which case B-scan can be helpful for studying the details of posterior segment [9]. In the Endophthalmitis Vitrectomy study, ultrasonography was used to detect retinal or choroidal detachment in cases with opaque media [10]. The ultrasound features of infectious endophthalmitis were first described by Oksala in three patients with vitreous abscess [11]. Ossoinig. described the occurrence of retino-choroidal thickening and vitreous membranes in severe endophthalmitis [12]. Chan., et al. reported the presence of anterior vitreous opacities and retinochoroidal thickening in all the studied 11 patients [13]. Thakker., et al. have reported the ultrasound abnormalities found in 54 patients with endophthalmitis which predominantly included severe vitreous debris, moderate and severe membranous debris and loculated pockets of vitreous debris. They noted choroidal thickening in $16.7 \%$, CD in 14.8\%, and RD in $7.4 \%$ of the eyes. More than one-fifth of the patients had retinal and choroidal detachments in that study [14]. In our study, 5.6\% of patients had RD and 1.9\% patients had CD. This difference may be due to the fact that they included all types of endophthalmitis.

The correlation of ultrasound features with visual outcome and microbiology was studied in the past (Table 4). Dense vitreous opacities were associated with poor presenting vision in our study, which is in concordance with the study by Dacey., et al [4]. They also reported an association between dense vitreous membranes, retinal, choroidal detachment, and poor final vision. Similarly, Rachitskaya., et al. had described an association between dense vitreous membranes, RD, CD and poor final vision [5]. Our study found a statistically significant association between presence of retinal detachment and poor final vision. In a study by Patil., et al. Staphylococcal epidermidis was most common isolated organism, cultured in 8 (33.4\%) out of 28 eyes. They found an association between dense vitreous opacities and virulent organisms like Staphylococcus aureus and Pseudomonas [15]. Coagulase negative staphylococci was the most common organism isolated in our study (18.5\%). The presence of retinal detachment and retino-choroidal thickening had a significant association with virulent organisms in our study.

\begin{tabular}{|c|c|c|c|}
\hline Study & Type of study and study period & Number of patients included & Association with poor final vision \\
\hline \multirow{5}{*}{ Dacey., et al. } & \multirow{5}{*}{ Retrospective, 1981-1992 } & \multirow{5}{*}{136} & Dense vitreous opacities (P-0.007) \\
\hline & & & Vitreous membranes $(\mathrm{P}=0.06)$ \\
\hline & & & $\mathrm{RD}(\mathrm{P}-0.003)$ \\
\hline & & & Extent of RD $(P=0.004)$ \\
\hline & & & $\mathrm{CD}(\mathrm{P}=0.002)$ \\
\hline \multirow{4}{*}{ Aleksandra., et al. } & \multirow{4}{*}{ Retrospective, 1996-2010 } & \multirow{4}{*}{149} & Dense vitreous opacities (P-0.005) \\
\hline & & & Marked membranes $(\mathrm{P}=0.0001$ \\
\hline & & & $\mathrm{RD}(\mathrm{P}<0.0001)$ \\
\hline & & & $\mathrm{CD}(\mathrm{P}<0.0001)$ \\
\hline Patil., et al. & Prospective, 1997-1999 & 28 & Dense vitreous opacities (P-0.03) \\
\hline Present Study & Prospective, 2013-2015 & 54 & $\mathrm{RD}(0.004)$ \\
\hline
\end{tabular}

Table 4: Comparison of final outcome by ultrasonographic features in previous studies and present study.

\section{Limitation of the Study}

The limitations in our study include smaller sample size and subjective grading of ultrasound features with varied gain settings. The inter observer variations may introduce potential errors in interpretation. Further trials might be needed to prove its utility in acute endophthalmitis using standardized ultrasonographic features and constant gain settings.

\section{Conclusion}

Ultrasonographic features at the initial presentation can be much useful in guiding the management of endophthalmitis, par-

Citation: Tharini Senthamizh., et al. "Sonographic Features and its Association with Visual Outcome and Microbiological Profile in Acute Post Cataract Surgery Endophthalmitis - A Prospective Analysis”. Acta Scientific Ophthalmology 3.10 (2020): 47-52. 
ticularly in patients with reduced media clarity and severe endophthalmitis caused by virulent organisms. The presence of retinal detachment and retinochoroidal thickening in ultrasound can alert the clinicians to proceed with aggressive treatment even before the arrival of culture results.

\section{Conflict of Interest}

None declared.

\section{Bibliography}

1. Somani S., et al. "Postoperative endophthalmitis: incidence, predisposing surgery, clinical course and outcome". Canadian Journal of Ophthalmology 32.5 (1997): 303-310.

2. Lalitha Prajna., et al. "A literature review and update on the incidence and microbiology spectrum of post cataract surgery endophthalmitis over past two decades in India". Indian Journal of Ophthalmology 65.8 (2017): 673-677.

3. Oksala A. "The echogram in vitreous opacities". American Journal of Ophthalmology 49 (1960): 1301-1307.

4. Dacey M P., et al. "Echographic findings in infectious endophthalmitis". Archives of Ophthalmology 112.10 (1994): 13251333.

5. Rachitskaya Aleksandra V., et al. "Correlation between baseline echographic features of endophthalmitis, microbiological isolates, and visual outcomes". Clinical Ophthalmology 7 (2013): 779-785.

6. Grover S., et al. "Visual acuity impairment in patients with retinitis pigmentosa at age 45 years or older". Ophthalmology 106.9 (1999): 1780-1785.

7. Cornut Pierre-Loïc., et al. "Relationship between baseline clinical data and microbiologic spectrum in 100 patients with acute postcataract endophthalmitis". Retina 32.3 (2012): 549557.

8. Sadaka Ama., et al. "Bacterial endophthalmitis in the age of outpatient intravitreal therapies and cataract surgeries: hostmicrobe interactions in intraocular infection". Progress in Retinal and Eye Research 31.4 (2012): 316-331.

9. Kohanim Sahar., et al. "Utility of ocular ultrasonography in diagnosing infectious endophthalmitis in patients with media opacities". Seminars in Ophthalmology 27.5-6 (2012): 242-245.

10. Wisniewski SR., et al. "Characteristics after cataract extraction or secondary lens implantation among patients screened for the Endophthalmitis Vitrectomy Study". Ophthalmology 107.7 (2000): 1274-1282.
11. Oksala A. "The echogram in vitreous opacities". American Journal of Ophthalmology 49 (1960): 1301-1307.

12. Ossoinig KC. "Standardized echography: basic principles, clinical applications, and results". International Ophthalmology Clinics 19.4 (1979): 127-210.

13. Chan IM., et al. "Ultrasonographic findings in endophthalmitis". Annals of Ophthalmology 16.8 (1984): 778-784.

14. Thakker MM., et al. "Ultrasonographic Characteristics of Eyes with Endophthalmitis". Investigative Ophthalmology and Visual Science 44.13 (2003): 1847.

15. Patil RTD., et al. "Relationship of echographic findings with visual outcomes in post-cataract surgery endophthalmitis". Annals of Ophthalmology 36 (2004): 12-16.

\section{Assets from publication with us}

- Prompt Acknowledgement after receiving the article

- Thorough Double blinded peer review

- Rapid Publication

- Issue of Publication Certificate

- High visibility of your Published work

Website: www.actascientific.com/

Submit Article: www.actascientific.com/submission.php

Email us: editor@actascientific.com

Contact us: +919182824667

Citation: Tharini Senthamizh., et al. "Sonographic Features and its Association with Visual Outcome and Microbiological Profile in Acute Post Cataract Surgery Endophthalmitis - A Prospective Analysis". Acta Scientific Ophthalmology 3.10 (2020): 47-52. 